\title{
NOTA SULLA NON LINEARITÀ DEL SISTEMA RESPIRATORIO: COME L'EMOGLOBINA LEGA GASTON TISSANDIER A REINHOLD MESSNER
}

\author{
Nota del s.c. GUIDO FERRETTI (*)
}

(Adunanza del 24 gennaio 2019)

SuNTO. - In questo articolo si prende spunto da una tragedia aeronautica per tracciare sommariamente la storia della curva di dissociazione dell'emoglobina per l'ossigeno e della creazione del modello di Hill. Dopo aver argomentato che le caratteristiche di tale curva causano il comportamento non lineare del sistema respiratorio, vengono discusse alcune conseguenze funzionali di tale comportamento, che spiegano come fu possibile che Reinhold Messner sia riuscito a compiere la straordinaria imprevista impresa di scalare la vetta dell'Everest senza ossigeno supplementare.

$$
* * *
$$

ABSTRACT. - This article starts from an aeronautical tragedy to track briefly the history of the oxygen equilibrium curve and the creation of Hill's model of it. After having argued that the characteristics of such a curve determine the non-linear behaviour of the respiratory system, I discussed some functional consequences of this behaviour, which explain how it happened that Reinhold Messner succeeded in the extraordinary enterprise of climbing Mount Everest without supplementary oxygen.

\section{UN DRAMMATICO INCIDENTE}

La tragedia aeronautica che avvenne nei cieli di Parigi il 15 aprile 1875 (Fig. 1) fece grande scalpore in Francia. Essa costò la vita

(") Dipartimento di Medicina Molecolare e Traslazionale, Università di Brescia, Italy. E-mail: guido.ferretti@unibs.it 
a due dei tre aviatori che tentavano un'ascesa in mongolfiera ad alta quota, mentre il terzo, il pilota, Gaston Tissandier (1843-1899), che pure perse conoscenza, rinvenne durante la discesa e riuscì a pilotare l'atterraggio. L'altimetro a bordo indicò il raggiungimento della quota di 8600 metri. Lo scalpore dipese anche dalla rilevanza scientifica del volo e dal coinvolgimento dell'Académie de France. Prima del volo, gli avieri erano stati studiati nella camera ipobarica costruita dal celebre fisiologo e accademico Paul Bert (1833-1886), che giudicò il tentativo fattibile, benché avesse suggerito la presenza a bordo di fiasche di ossigeno dotate di boccaglio, da usare in caso di necessità. Ovviamente, i tre svennero prima di riuscire ad afferrare la fiasca.

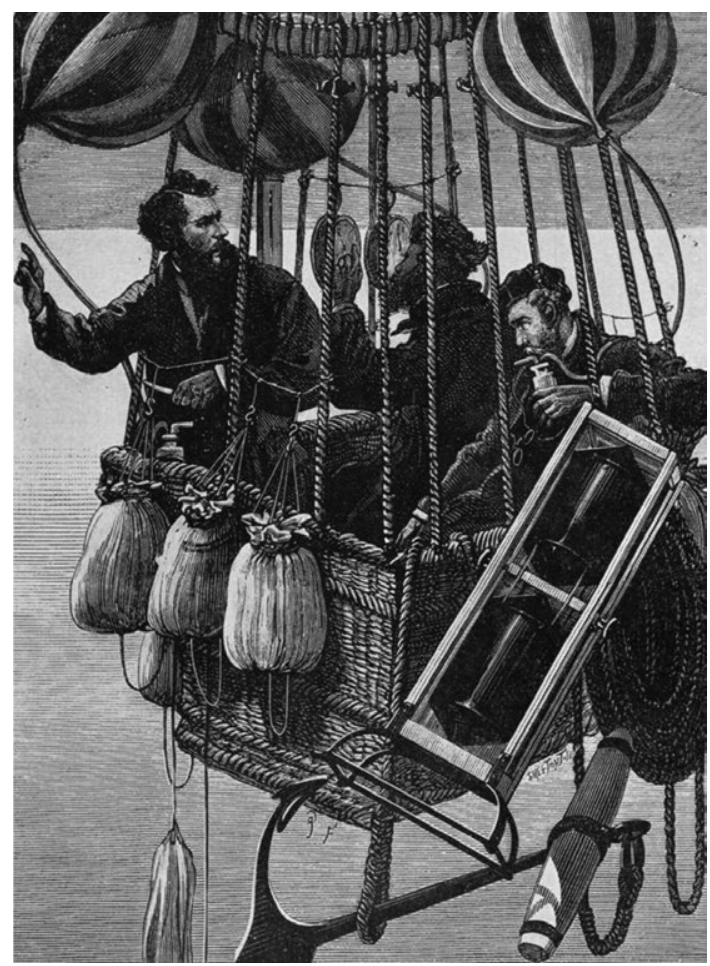

Fig. 1. Un'immagine del volo in pallone aerostatico del 15 aprile 1875, a Parigi, prima dell'incidente. Gaston Tissandier, capitano della navicella, unico sopravvissuto, è l'uomo a sinistra. Sul davanti della cesta si vedono le fiasche contenenti ossigeno, che Paul Bert volle venissero portate a bordo. 
Paul Bert fu incaricato di studiare le cause della morte dei due avieri. I suoi primi risultati furono resi pubblici tre anni dopo, all'interno del suo celeberrimo volume intitolato La Pression Barométrique [1]. Egli infatti riuscì a misurare la concentrazione di ossigeno nel sangue di uomini esposti a gradi diversi di ipossia e costruì un diagramma (Fig. 2) in cui pose la concentrazione di ossigeno nel sangue in funzione della pressione parziale di ossigeno nell'aria inspirata. Egli dimostrò così che quella diminuiva in funzione di questa e concluse che la causa della morte degli avieri fu la scarsità di ossigeno. Il diagramma costruito da Bert appariva però sorprendente: benché la pressione parziale di ossigeno calasse più o meno in proporzione con la pressione barometrica, cioè con l'altitudine sul livello del mare, la diminuzione della concentrazione di ossigeno nel sangue non avveniva in proporzione ad esse, ma era modesta ad elevate pressioni parziali nell'aria inspirata, per divenire molto più intensa a basse pressioni parziali. Un elemento di non-linearità interferiva, ma quale?

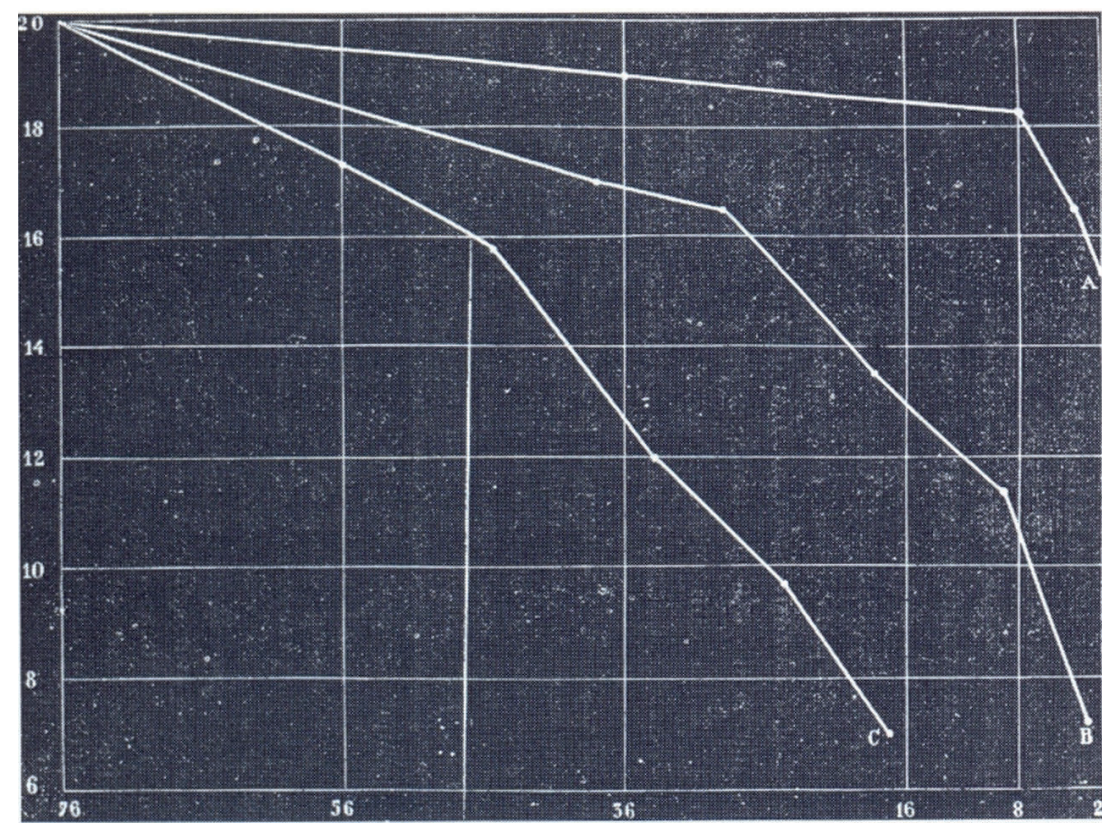

Fig. 2. La primitiva curva di dissociazione dell'emoglobina per l'ossigeno, costruita da Paul Bert a partire da dati di concentrazione e pressione parziale di ossigeno ottenuti in camera ipobarica, all'Académie de France. 


\section{UNO STRANO PIGMENTO}

Negli stessi anni, Felix Hoppe-Seyler (1825-1895), a Tubinga, isolò il pigmento del sangue, che egli chiamò emoglobina, e dimostrò che esso legava l'ossigeno. Alcuni anni dopo, a Copenhagen, Christian Bohr (1855-1911), al quale in séguito per questo fu assegnato il Premio Nobel per la Fisiologia o la Medicina, espose soluzioni di emoglobina a diverse pressioni parziali di ossigeno, costruendo così quella che oggi consideriamo la prima curva di dissociazione dell'emoglobina per l'ossigeno [2]: una curva quindi, non una retta, orizzontale ad elevata pressione parziale, e progressivamente più ripida man mano che la pressione parziale di ossigeno calava, alla quale Bohr diede una soluzione iperbolica. Ecco una possibile risposta al quesito di Bert sull'elemento di non-linearità. Fu quindi il successore di Hoppe-Seyler a Tubinga, Carl Gustaf von Hüfner (1840-1908), a sostenere la correttezza del risultato di Bohr anche sul piano teorico, in quanto compatibile con la legge di azione di massa per la reazione:

$\mathrm{Hb}+\mathrm{O}_{2} \leftrightarrow \mathrm{HbO}_{2}$

Il trattamento che Bohr e von Hüfner diedero della curva di dissociazione dell'emoglobina per l'ossigeno era simile a quello che successivamente Leonor Michaelis (1875-1949) e Maud Menten (1879-1960) avrebbero dato alle cinetiche enzimatiche.

Fu Bohr stesso a confutare [3] la sua ipotesi, e prima che Michaelis e Menten pubblicassero la loro teoria. Egli infatti pubblicò la curva di dissociazione dell'emoglobina determinata sul sangue intero, e non su soluzioni di emoglobina pura, dimostrandone la forma sigmoidale: una forma incompatibile sul piano teorico con l'ipotesi primigenia. L'anno successivo Bohr, coi suoi allievi Karl Albert Hasselbalch (1874-1962) e August Krogh (1874-1949), dimostrò che composti terzi (nel caso, il diossido di carbonio) avevano il potere di alterare la forma della curva di dissociazione dell'emoglobina per l'ossigeno [4], come mostrato in Fig. 3. In memoria del vecchio maestro, il fenomeno è stato tramandato sotto il nome di effetto Bohr. Fu John Barcroft (1872-1947) qualche anno dopo, a dimostrare che le alterazioni di forma della curva di dissociazione dovute all'effetto Bohr dipendevano dall'aggiunta o sottrazione di acidi [5]. Non fu però Barcroft, bensì il suo allievo Archibald Vivian Hill (1886-1977) 
a dare una soluzione matematica elegante al concetto dell'effetto Bohr.

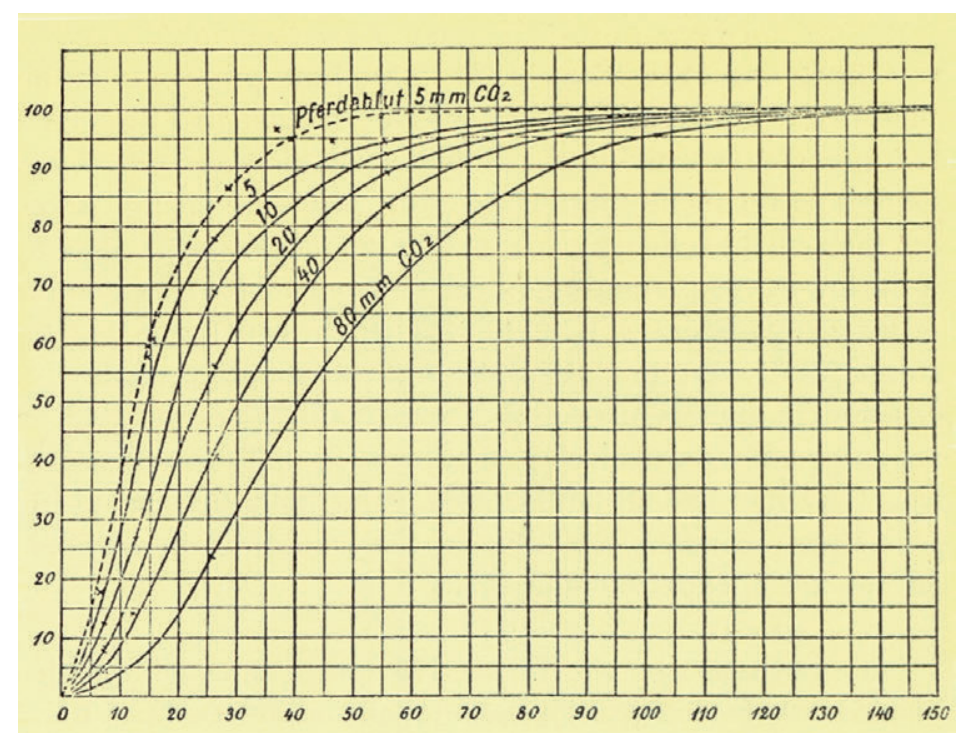

Fig. 3. Curve di dissociazione dell'emoglobina per l'ossigeno ottenute da Christian Bobr su sangue intero durante esposizione a diverse pressioni parziali di anidride carbonica. È questa la prima rappresentazione grafica dell'effetto Bobr.

\section{Il mOdello E la CONGETTURA Di HiLl}

Hill [6] propose che la curva di dissociazione dell'emoglobina riflettesse l'equilibrio della seguente reazione chimica tra emoglobina e ossigeno:

$\mathrm{Hb}+\mathrm{n}_{2} \leftrightarrow \mathrm{Hb}\left(\mathrm{O}_{2}\right)_{\mathrm{n}}$

dove $\mathrm{n}$ indica il rapporto stechiometrico della reazione. Questa formulazione differisce da quella dell'equazione (1) per un piccolo dettaglio, però importante: questa ammette $\mathrm{n}>1$, quella no. Si noti che allora non si conosceva il valore di n, essendo la struttura chimica dell'emoglobina ancora ignota. Con la formulazione di Hill, la costante di equilibrio della reazione $(\mathrm{K})$ è: 
$\mathrm{K}=\frac{\left[\mathrm{HbO}_{2}\right]^{\mathrm{n}}}{\left[\mathrm{Hb}-\left[\mathrm{O}_{2}\right]^{n}\right.}$

dove $\mathrm{HbO}_{2}$ e $\mathrm{Hb}^{-}$indicano rispettivamente l'emoglobina ossigenata e l'emoglobina non ossigenata (ridotta). Essendo l'emoglobina totale la somma di $\mathrm{HbO}_{2}$ e $\mathrm{Hb}^{-}$, possiamo definire la saturazione $(S)$ dell'emoglobina per l'ossigeno come:

$$
S=\frac{\left[\mathrm{HbO}_{2}\right]}{\left[\mathrm{HbO}_{2}\right]+\left[\mathrm{Hb}^{-}\right]}
$$

Considerato che pressoché tutto l'ossigeno presente nel sangue, in condizioni fisiologiche, sta legato all'emoglobina, e che $\mathrm{HbO}_{2}$ sta in equilibrio con la pressione parziale di ossigeno $\left(\mathrm{PO}_{2}\right)$, Hill giunse, con una serie di passaggi algebrici successivi, alla seguente formulazione:

$$
\frac{S}{(1-S)}=\Sigma=\left(\frac{P O_{2}}{P_{k}}\right)^{n}
$$

dove $P_{k}$ è una costante. Esprimendo l'equazione (5) in forma logaritmica, si ha:

$$
\log \Sigma=n\left(\log \mathrm{PO}_{2}-\log P_{k}\right)
$$

Pertanto, se mettiamo $\log \Sigma$ in funzione di $\log \mathrm{PO}_{2}$ (Fig. 4), otteniamo relazioni lineari, in cui il coefficiente angolare è uguale a $n$ e l'intercetta sulla ascisse è uguale a $\log P_{k}$. Per quanto riguarda $n$, Hill congetturò che esso corrispondesse all'equilibrio stechiometrico della reazione descritta dall'equazione (2), cioè al numero di molecole di ossigeno in grado di legarsi a una molecola di emoglobina. Egli ottenne $n=1$ per la mioglobina (il pigmento, simile all'emoglobina, che troviamo nelle fibre muscolari) e $n=2.8$ per l'emoglobina. Inoltre, nella rappresentazione grafica dell'equazione (6), le curve di dissociazione dell'emoglobina riprodotte in Fig. 3, che Bohr ottenne variando la concentrazione (o la pressione parziale) di $\mathrm{CO}_{2}$, si traducono in una famiglia di rette parallele ( $n$ invariante ed indipendente da tutti gli altri termini dell'equazione 6), spostate progressivamente verso destra man mano che la concentrazione di $\mathrm{CO}_{2}$, e quindi $\log P_{k}$, aumenta. Da qui deriva il concetto di spostamen- 
to a destra (o a sinistra) della curva di dissociazione dell'emoglobina per l'ossigeno, di cui ancora oggi si parla agli studenti e di cui molto si abusa in àmbito clinico. Per parte sua, il valore di $\log P_{k}$ corrisponde a quello che $\log \mathrm{PO}_{2}$ assume quando $\log \Sigma$ è uguale a 0 , e quindi $\Sigma$ è uguale a 1. Ciò si verifica, secondo l'equazione (5), quando $S=0.5$. Pertanto Hill definì la costante $P_{k}$ come $P_{50}$, vale a dire la $P_{2}$ corrispondente a $S=0.5$. Un aumento di $P_{50}$ corrisponde sempre a uno spostamento a destra della curva di dissociazione dell'emoglobina per l'ossigeno. Questi concetti sono validi solo all'interno del modello di Hill, e quindi parlare di «spostamento a destra» o di $P_{50}$ comporta accettazione implicita del modello all'interno dei quali sono stati creati.

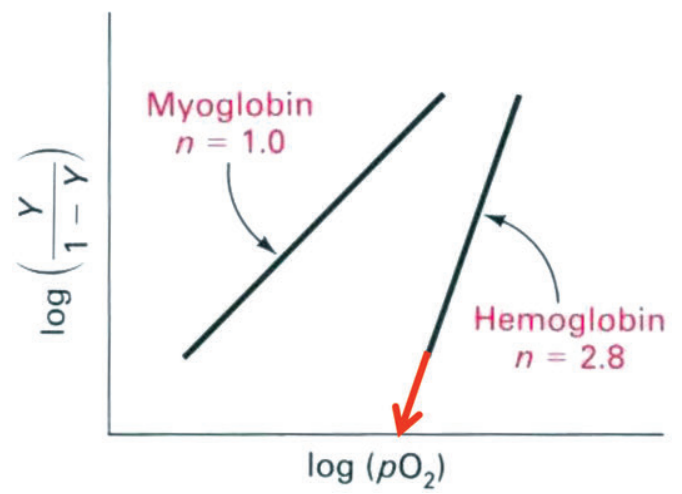

Fig. 4. Rappresentazione semilogaritmica del modello di Hill della curva di dissociazione dell'emoglobina per l'ossigeno, con indicazione della pendenza n, che Hill congetturò corrispondesse al rapporto stechiometrico tra ossigeno ed emoglobina, per la mioglobina $(\mathrm{n}=1.0) e$ per l'emoglobina $(\mathrm{n}=2.8)$. Il secondo valore è indicativo di cooperatività. La freccia indica l'intercetta sull'asse delle ascisse, corrispondente a quella che Hill chiamò $\mathrm{P}_{50}$.

\section{Confutazione E CRITICA DELla CONGETTURA Di Hill}

La confutazione della congettura di Hill giunse quando Max Perutz (1914-2002) delucidò la struttura quaternaria dell'emoglobina, per cui ottenne il Premio Nobel per la Chimica nel 1962. Egli dimostrò che ogni molecola di emoglobina, composta di quattro apoproteine e quattro gruppi prostetici, era in grado di legare fino a quattro molecole di ossigeno [7], per cui $n=4$ anziché 2.8. Per la mioglobina fu invece confermato $n=1$. Dopo la confutazione della congettura di Hill, il mondo della fisiologia 
respiratoria tese ad abbandonare il suo modello della curva di dissociazione, giudicandolo falso. Modelli teorici alternativi non furono però proposti. I pedanti, che non mancano mai, applicando anche tecniche analitiche sempre più precise, definirono modelli empirici polinomiali della curva di dissociazione dell'emoglobina sempre più complessi. Basta aumentarne $\mathrm{i}$ gradi, si troveranno sempre polinomi in grado di descrivere un andamento con precisione sempre maggiore. Tutti questi modelli, però, non avevano alcun legame teorico con modelli fisiologici soggiacenti: essi furono tutti esclusivamente descrittivi ed ebbero un impatto culturale infimo.

Benché confutato sul piano quantitativo riguardo al valore di $n$, il modello di Hill aveva un legame concettuale solidissimo con una realtà fisiologica soggiacente, forniva uno strumento di analisi quantitativa molto potente dell'effetto Bohr, e gli stessi pedanti lo considerano un descrittore sufficientemente preciso della curva di dissociazione, almeno nell'àmbito compreso tra $S=0.2$ e $S=0.98$. Credo che, invece di abbandonarlo come falso, sarebbe stato più prudente rivisitarlo, correggendone il significato, dopo la confutazione di Perutz: $n=1$ (mioglobina, una sola apoproteina, con un solo gruppo prostetico) significa che le molecole di ossigeno si legano all'emoglobina in maniera indipendente l'una dall'altra; $n>1$ significa presenza di cooperatività, che si ha quando le apoproteine (e i gruppi prostetici) sono più di uno, e quindi la proteina assume struttura quaternaria. Hill applicò l'equazione logaritmica all'intera gamma di valori di $S$ : ciò comportò una distorsione della retta a causa dell'impatto dei valori di $S<0.2$. La curva di dissociazione è in effetti sigmoide, causa allosteria. Pertanto il valore di 2.8 per $n$, riportato da Hill, potrebbe derivare dal fatto che l'emoglobina ha una difficoltà maggiore a tenere l'ossigeno legato quando predomina nettamente la forma ridotta.

E' comunque un fatto che il modello di Hill, o un altro modello teorico ad esso alternativo, è necessario per comprendere gli effetti che la curva di dissociazione dell'emoglobina per l'ossigeno ha sul comportamento non lineare del sistema respiratorio, e le conseguenze fisiologiche che da ciò derivano.

\section{COMPORTAMENTO NON LINEARE DEL SISTEMA RESPIRATORIO}

Oggi si guarda al sistema respiratorio come a un sistema integrato in cui un gas fluisce lungo il percorso dalla bocca ai mitocondri (o viceversa) sospinto da un gradiente di pressione, necessario a vincere le 
numerose resistenze in serie che si oppongono al flusso. La Fig. 5 evidenzia le resistenze (o il loro reciproco, le conduttanze) identificate a varî livelli lungo il sistema e riporta le relative equazioni di conduttanza locali. La resistenza totale in un tale sistema è data dalla somma di tutte le resistenze parziali. Ogni resistenza costituisce quindi una frazione della resistenza totale al flusso di gas. In un sistema lineare, la frazione di resistenza totale rappresentata da ciascuna resistenza parziale è calcolabile semplicemente dal rapporto tra il gradiente di pressione che sostiene il flusso di gas a un dato livello e il gradiente di pressione totale lungo l'intero sistema. Questo principio vale per tutti i gas che nel sangue sono presenti solo in forma fisicamente disciolta, o la cui curva di dissociazione è, nell'àmbito di pressioni parziali considerate, lineare. Questo principio non vale però per il flusso di ossigeno, a causa della componente di non linearità introdotta dalla curva di dissociazione dell'emoglobina: l'equazione di conduttanza per l'ossigeno deve avere soluzione non lineare, qualunque modello si voglia applicare alla curva di dissociazione.

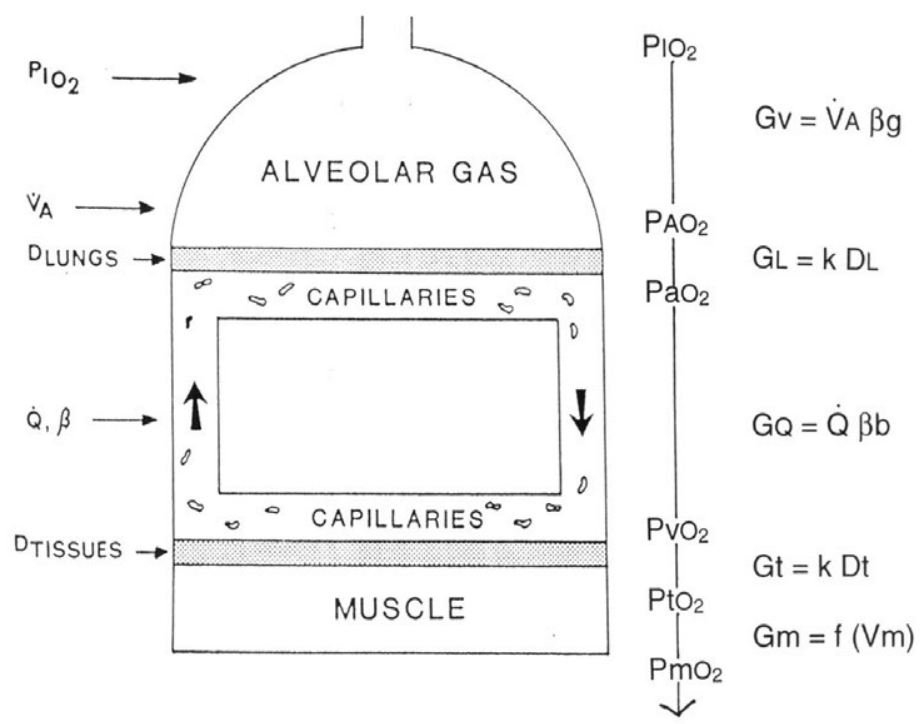

Fig. 5. Rappresentazione schematica del sistema respiratorio, con identificazione sia dei siti dove è possibile misurare la pressione parziale di ossigeno, sia delle diverse conduttanze di significato funzionale lungo il sistema, disposte in serie. Per ogni conduttanza, è indicata la relativa equazione di conduttanze. Di queste conduttanze, due (la conduttanza ventilatoria e la conduttanza cardiovascolare) risultano dal prodotto di due variabili fisiologiche, le altre sono proporzionali a variabili fisiologiche. 
La prima soluzione non lineare dell'equazione di conduttanza dell'ossigeno fu proposta dal canadese Roy Shephard [8], ma passò rapidamente nell'oblio. Il principio però covò sotto la cenere del rogo delle idee e fu ripreso da Dick Taylor di Boston e Ewald Weibel di Berna come base teorica per il loro celebrato studio morfometrico sul sistema respiratorio dei mammiferi [9]. Da qui partì il socio corrispondente Pietro Enrico di Prampero per creare il primo modello multifattoriale dei fattori limitanti il massimo consumo di ossigeno nell'uomo all'esercizio [10], con cui egli riuscì a dimostrare l'inapplicabilità del principio di linearità al sistema respiratorio, e a stimare, in virtù di questo, che il $70 \%$ della limitazione del massimo consumo di ossigeno fosse da attribuirsi al trasporto cardiovascolare di ossigeno, anziché il 50\% che si ottiene assumendo comportamento lineare del sistema. Tale stima ricevette poi solido sostegno sperimentale da parte di un gruppo coordinato dal sottoscritto [11].

\section{EQUAZIONE DI CONDUTTANZA PER L'OSSIGENO A LIVELLO CARDIOVASCOLARE E MECCANISMO DELLE RISPOSTE NON LINEARI}

Di Prampero e Ferretti [12] inferirono sul meccanismo con cui la non linearità della curva di dissociazione dell'emoglobina esplicava i suoi effetti sul massimo flusso di ossigeno $\left(\mathrm{VO}_{2}\right.$ max $)$ nel sistema respiratorio. Ammettiamo di ridurre improvvisamente e acutamente la resistenza al flusso di ossigeno nelle vie aeree, per esempio facendo respirare una miscela di gas contenente elio al posto dell'azoto. Ciò comporta un immediato rialzo della pressione parziale di ossigeno nell'aria alveolare, e così a valle, fino al sangue arterioso. Nel sangue arterioso però la concentrazione di ossigeno, che pure varia con la pressione parziale dello stesso, è dettata dalla forma della curva di dissociazione dell'emoglobina.

Nel modello delle conduttanze in serie, $\mathrm{VO}_{2}$ max a livello cardiovascolare è descritto dalla seguente equazione:

$$
\dot{\mathrm{V}} \mathrm{O}_{2} \max =\dot{Q} \beta b\left(\mathrm{PaO}_{2}-\mathrm{P} \bar{v} \mathrm{O}_{2}\right)
$$

dove $\dot{Q}$ è il flusso ematico totale (gettata cardiaca) e $\beta b$ è una costante definita coefficiente di trasporto dell'ossigeno nel sangue, mentre il loro prodotto rappresenta la conduttanza. La costante $\beta b$ è uguale a: 


$$
\beta b=\frac{\mathrm{CaO}_{2}-\mathrm{C} \bar{v} \mathrm{O}_{2}}{\mathrm{PaO}_{2}-\mathrm{P} \bar{v} \mathrm{O}_{2}}
$$

in cui il numeratore indica la differenza di concentrazione di ossigeno tra sangue arterioso $(a)$ e sangue venoso misto $(\bar{v})$, mentre il denominatore corrisponde alla differenza di pressione parziale per il medesimo gas tra i due sangui. Come appare dalla Fig. 6, l'equazione (8) definisce $\beta b$ come pendenza media della curva di dissociazione dell'emoglobina per l'ossigeno.

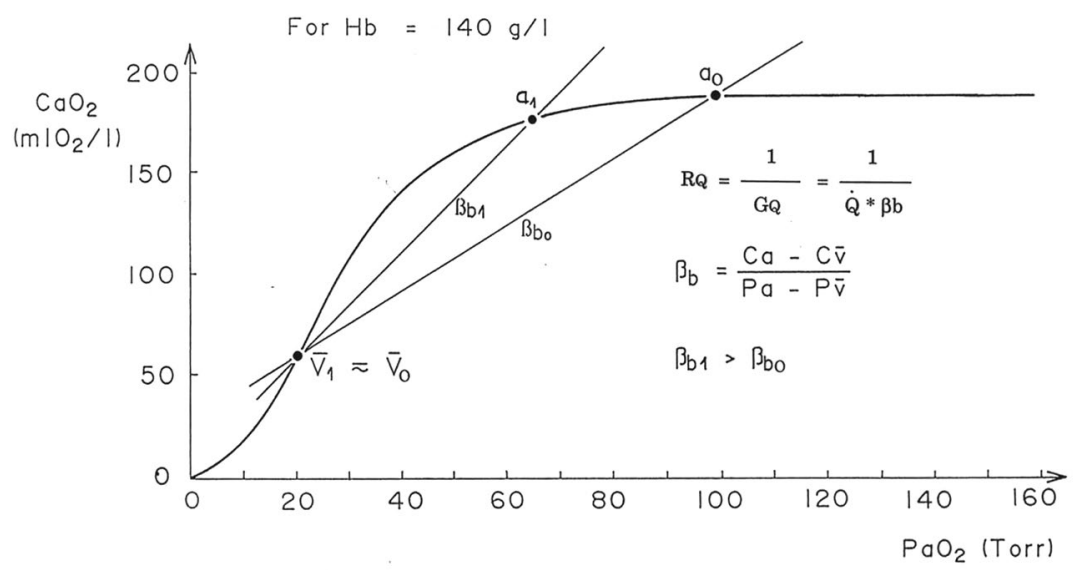

Fig. 6. Curva di dissociazione dell'emoglobina per l'ossigeno, con indicazione delle pendenze medie tra punto del sangue arterioso (a) e punto del sangue venoso misto (v), per casi in cui il punto a si situa sulla parte piatta della curva, oppure in prossimità della parte ripida. Sono indicate anche le definizioni di resistenza cardiovascolare $\left(R_{Q}\right)$ e del coefficiente di trasporto dell'ossigeno nel sangue $\left(\beta_{\mathrm{b}}\right)$.

Se $\mathrm{PaO}_{2}$ aumenta in séguito ad una variazione di conduttanza ventilatoria, a tale aumento non consegue però una variazione di $\mathrm{CaO}_{2}$, poiché a livello del mare operiamo sulla parte piatta della curva di dissociazione dell'emoglobina. Dal lato opposto, pressione parziale e concentrazione nel sangue venoso misto, se non variano, calano di poco. Pertanto, la pendenza media della curva, cioè $\beta b$, diminuisce. Ne conseguono la diminuzione della conduttanza e quindi l'aumento del suo reciproco, cioè la resistenza a livello cardiovascolare. 
Ci troviamo pertanto in una condizione in cui, a séguito della variazione indotta di una conduttanza, quella ventilatoria, a causa della riduzione di densità della miscela respirata, si verifica necessariamente anche una variazione opposta di una seconda conduttanza, quella cardiovascolare, che tende a compensare ed annullare gli effetti della prima. L'esito è che una diminuzione di resistenza a livello ventilatorio, che naturalmente tenderebbe ad aumentare il $\mathrm{VO}_{2} \max$, non ha invece alcun effetto sul $\mathrm{VO}_{2}$ max a livello del mare [13]. Questo autorizza a concludere che, a causa della non linearità del comportamento del sistema respiratorio, le resistenze in serie situate a monte del trasporto cardiovascolare di ossigeno non contribuiscono a limitare il $\mathrm{VO}_{2}$ max. È per questo che di Prampero [10] ottenne una limitazione cardiovascolare del $70 \%$ anziché del $50 \%$, come previsto da un modello lineare: egli si trovò ad analizzare un sistema dotato di solo due resistenze efficaci nel limitare il $\mathrm{VO}_{2} \max$, cioè in grado di ripartirsi la limitazione totale.

\section{Massimo Consumo di OsSigeno in ALtitudine}

Il $\mathrm{VO}_{2}$ max diminuisce in altitudine a causa della diminuzione della pressione barometrica. Numerosi studiosi, tra cui alcuni membri di questa Accademia [14, 15], riportarono, in circostanze diverse, che la caduta del $\mathrm{VO}_{2}$ max in altitudine è marcatamente non lineare: modesta a quote inferiori a 2000 metri, molto accentuata a quote superiori a 4000 metri.

La caduta del $\mathrm{VO}_{2}$ max in ipossia è rappresentata in Fig. 7 per individui aventi a livello del mare un massimo consumo di ossigeno equivalente a quello medio della popolazione di sesso maschile non atletica [16]. Tale rappresentazione, teorica ma basata su una grande mole di dati sperimentali, equivale alla costruzione di una curva flusso-pressione: le rette convergenti sull'origine degli assi sono isoplete di conduttanza. Appare così chiaro che la non linearità della caduta del $\mathrm{VO}_{2}$ max in altitudine comporta un aumento della conduttanza totale del sistema respiratorio. Tale aumento appare come una conseguenza di un progressivo incremento di $\beta b$, cioè della conduttanza cardiovascolare [17]. 


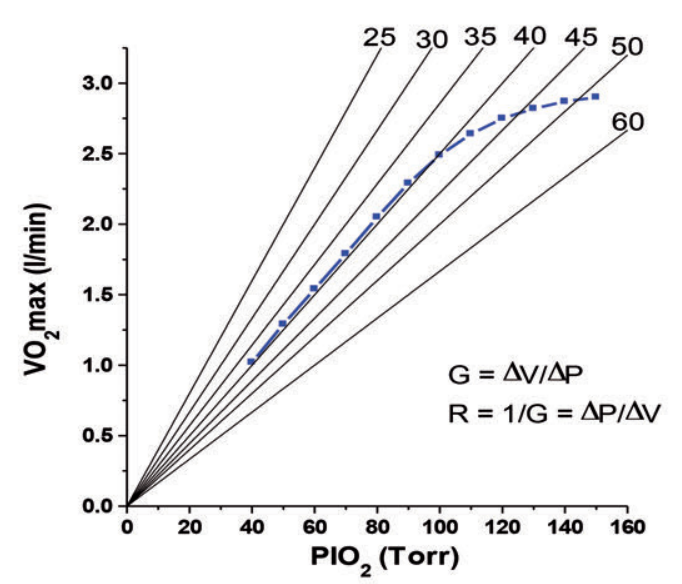

Fig. 7. La curva blu dimostra l'andamento della caduta del massimo consumo di ossigeno in ipossia. Le linee sottili nere, convergenti sull'origine degli assi, sono isoplete di conduttanza (o del reciproco di questa, la resistenza). Per ogni isopleta è indicato il corrispondente valore di resistenza. La forma della curva comporta calo di resistenza al flusso di ossigeno con il progressivo diminuire della pressione parziale di ossigeno nell'aria inspirata.

Ad altitudini inferiori ai 2000 metri, operiamo sulla parte piatta della curva di dissociazione dell'emoglobina per l'ossigeno. Pertanto ogni diminuzione della pressione barometrica, a cui si associa una diminuzione della $\mathrm{PaO}_{2}$, non è seguita da una diminuzione della $\mathrm{CaO}_{2}$. Così $\beta b$ assume un valore progressivamente più elevato, che fa crescere la conduttanza cardiovascolare, riducendo pertanto la corrispettiva resistenza. Ciò mantiene il $\mathrm{VO}_{2} \max$ più alto di quello che ci dovremmo attendere ad ogni altitudine in caso di comportamento lineare del sistema. Il fenomeno è tanto più accentuato quanto più intense sono le risposte ventilatorie all'ipossia [18]. È solo quando, salendo, si giunge ad operare sulla parte pendente della curva di dissociazione che la caduta del $\mathrm{VO}_{2}$ max diviene importante e tende a linearizzarsi ( $\beta b$ invariante). Wagner [19] dimostrò che il segmento di curva di dissociazione su cui si opera alle altitudini più estreme sulla Terra è a tutti i fini pratici indistinguibile da un segmento di retta. È pertanto verosimile che i due avieri del 1875 siano periti per aver raggiunto, in alta quota, una $\mathrm{CaO}$ incompatibile con una sufficiente ossigenazione cerebrale, ma se ciò avvenne, fu ad una altitudine ben più elevata di quanto non ci si potesse immaginare allora. 


\section{AtLETI IN ALTITUDine}

Dempsey et al [20] furono i primi a riportare che atleti caratterizzati da elevati valori di $\mathrm{VO}_{2} \max$ presentano desaturazione del sangue arterioso durante esercizio massimale a livello del mare. Questi autori attribuirono il fenomeno al fatto che tali atleti raggiungono anche valori di gettata cardiaca massima molto elevati, fino a $401 \mathrm{~min}^{-1}$, tali per cui il tempo di transito attraverso i capillari polmonari si riduce al punto di non consentire un'ossigenazione completa del sangue capillare.

$\mathrm{Nel}$ presente contesto tale osservazione assume valore in quanto ne consegue che durante esercizio massimale a livello del mare gli atleti operano già in prossimità della parte ripida della curva di dissociazione dell'emoglobina per l'ossigeno. Pertanto in essi il meccanismo, legato alle caratteristiche della curva di dissociazione, che mantiene il elevato a quote inferiori ai 2000 metri, non può funzionare.

$\mathrm{Ne}$ deriva che il $\mathrm{VO}_{2}$ max di atleti deve diminuire in altitudine in misura maggiore di quanto avviene in individui non atletici, a causa di un minor aumento della conduttanza cardiovascolare. Questa ipotesi, che ha ricevuto solida conferma sperimentale in ipossia acuta [21], ha come corollario che le naturali differenze di $\mathrm{VO}_{2} \max$ tra la popolazione si riducono progressivamente e consistentemente col salire dell'altitudine, fino quasi ad annullarsi alla quota corrispondente alla cima dell'Everest.

\section{Messner RagGiunge la Cima Dell'Everest SENZA OssigenO SUPPLEMENTARE}

Il giorno 8 maggio 1978 è una data cardine della storia non solo dell'alpinismo, ma di tutta l'esplorazione della Terra da parte dell'uomo: per la prima volta due alpinisti, Reinhold Messner e Peter Habeler, raggiunsero la vetta più alta della Terra, il Monte Everest (8848 metri sopra il livello del mare) senza fare uso di bombole contenenti ossigeno supplementare. Il mondo della scienza riteneva l'impresa impossibile, anche se in realtà qualcuno aveva espresso per iscritto e in tempi non sospetti opinioni diverse ma neglette [22]. Messner rivoluzionò l'alpinismo himalayano e tracciò una via nuova all'esplorazione montana presto percorsa anche da altri. Al fine di capire meglio cosa avvenne o come l'impresa fu possibile, Paolo Cerretelli, oggi membro effettivo di 
questa Accademia, e Hans Hoppeler di Berna organizzarono un ampio studio di valutazione funzionale di alpinisti che salirono vette di altezza superiore a 8500 metri sul livello del mare [23], tutti seguiti come medico da Oswald Oelz di Zurigo. L'assioma era che questi alpinisti dovessero avere caratteristiche fisiologiche uniche, da cui derivò l'ipotesi che il loro $\mathrm{VO}_{2} \max$ fosse straordinariamente elevato a livello del mare. Questa ipotesi fu confutata sperimentalmente, tra lo sconcerto generale: il $\mathrm{VO}_{2} \max$ degli alpinisti investigati era di gran lunga inferiore a quello degli atleti (Fig. 8). Non sapendo cosa dire, si concluse l'articolo con affermazioni metafisiche sul ruolo che potrebbe aver avuto la motivazione psicologica all'impresa: allora, non si conoscevano ancora gli effetti dell'effetto Dempsey sulla caduta del massimo consumo di ossigeno in altitudine, lo studio di Dempsey era stato pubblicato da troppo poco tempo. Oggi si sarebbe concluso che non serve avere massimi consumi di ossigeno straordinariamente elevati per raggiungere la cima dell'Everest senza ossigeno supplementare, a causa dell'effetto Dempsey e dell'effetto della non linearità del sistema respiratorio: il legame tra la tragedia del 1875 e il trionfo del 1978 è completo.

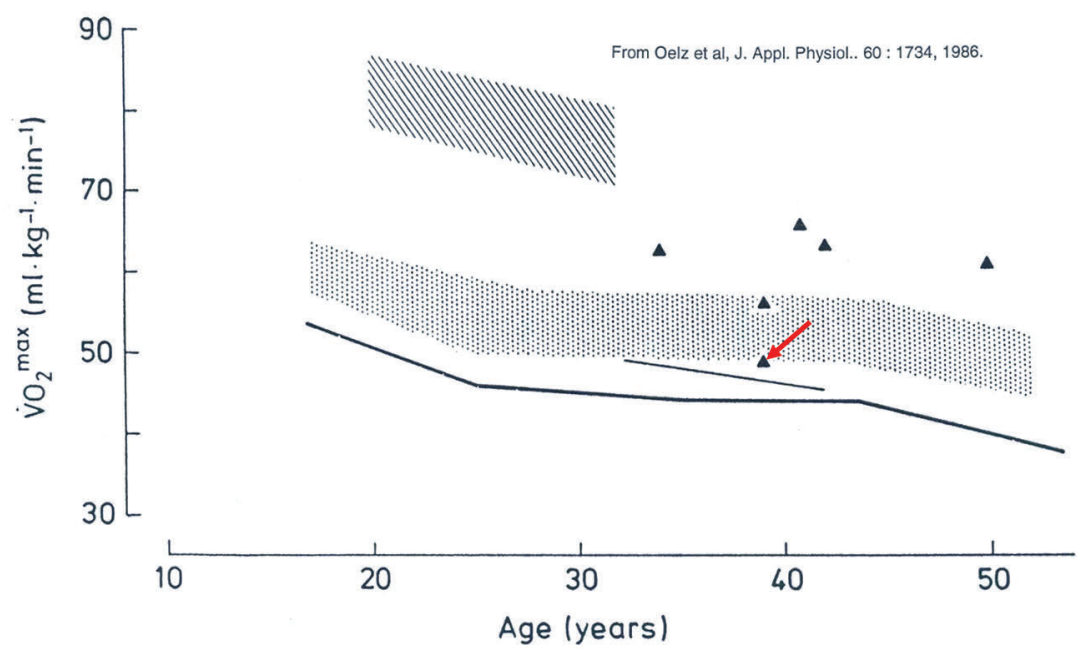

Fig. 8. Massimo consumo di ossigeno in funzione dell'età. La banda tratteggiata in alto a sinistra si riferisce ad atleti; la banda a puntini a non-atleti allenati. La traccia in basso definisce la normalità per il non atleta. I punti sperimentali riguardano sei alpinisti che banno asceso le più alte vette della Terra senza ossigeno supplementare. La freccia indica il valore di Reinhold Messner. 


\section{CONCLUSIONI}

Esplorazione umana e conoscenza fisiologica seguono spesso percorsi paralleli, che talora s'incrociano. Fu un clamoroso incidente mortale avvenuto a Parigi nel 1875 a dare il via a studi che condussero a definire le caratteristiche del legame tra ossigeno ed emoglobina ed in séguito a delineare il comportamento non lineare del sistema respiratorio e a identificare alcune delle conseguenze funzionali che questo avrebbe avuto. È attraverso un tale percorso che si sono costruiti gli strumenti culturali per comprendere il significato fisiologico di uno straordinario evento di un secolo dopo. Molti uomini di scienza furono protagonisti di questo percorso, che unisce le vicende diverse di due avventurosi, Tissandier e Messner. È per me motivo di fierezza constatare il ruolo importante di membri di questa Accademia nella costruzione di questo percorso.

\section{REFERENZE}

1. Bert P, La Pression Barométrique, Masson, Paris, 1878.

2. Bohr C, Experimentale Untersuchungen über die Sauerstoffaufnabme des Blutfarbstoffes, OC Olsen \& Co Buchdruckerei, Copenhagen, 1885.

3. Bohr C, Theoretische Behandlung der quantitativen Verhältnisse bei der Sauerstoffaufnahme der Hemoglobins, Zentralbl Physiol, 1903: 17: 682-691.

4. Bohr C, Hasselbalch KA, Krogh A, Über einen in biologischer Beziehung wichtigen Einfluss, den die Kohlensäurespannung des Blutes auf dessen Sauerstoffbindung übt, Skand Arch Physiol, 1904: 16: 402-414.

5. Barcroft J, The Respiratory Function of the Blood. Cambridge University Press, Cambridge, 1914.

6. Hill AV, The possible effects of the aggregation of the molecules of haemoglobin on its dissociation curves, J Physiol Lond 1910: 40: iv-vii.

7. Perutz MF, Stereochemistry of cooperative effects in haemoglobin, Nature 1970: 228: 726-733.

8. Shephard RJ, A non-linear solution of the oxygen conductance equation: applications to performance at sea level and at an altitude of $7350 \mathrm{ft}$, Int $Z$ angew Physiol, 1969: 27: 217-225.

9. Taylor CR, Weibel ER, Design of the mammalian respiratory system. I. Problem and strategy, Respir Physiol, 1981: 44: 1-10.

10. di Prampero PE, Metabolic and circulatory limitations to at the whole animal level, J Exp Biol, 1985: 115: 319-331.

11. Bringard A, Pogliaghi S, Adami A, De Roia G, Lador F, Lucini D, Pizzinelli P, Capelli C, Ferretti G, Cardiovascular determinants of maximal oxygen con- 
sumption in upright and supine posture at the end of prolonged bed rest in humans, Respir Physiol Neurobiol, 2010: 172: 53-62.

12. di Prampero PE, Ferretti G, Factors limiting maximal oxygen consumption in humans, Respir Pbysiol, 1990: 80: 113-128.

13. Esposito F, Ferretti G, The effects of breathing $\mathrm{He}-\mathrm{O}_{2}$ mixtures on maximal oxygen consumption in normoxic and hypoxic men. J Physiol Lond, 1997: 503: 215-221.

14. Cerretelli $\mathrm{P}$, Margaria R, Maximum oxygen consumption at altitude, Int $Z$ angew Physiol, 1961: 18: 460-464.

15. Cerretelli P, Gas exchange at high altitude, in: Pulmonary Gas Exchange, West JB editore, volume II, 97-147, Academic Press, New York, 1980.

16. Ferretti G, Energetics of Muscular Exercise, Springer, Heidelberg, 2015.

17. Ferretti G, Limiting factors to oxygen transport to Mount Everest 30 years after: a critique of Paolo Cerretelli contribution to altitude physiology, Eur J Appl Physiol, 2003: 90: 344-350.

18. Marconi C, Marzorati M, Grassi B, Basnyat B, Colombini A, Kayser B, Cerretelli P, Second generation Tibetan lowlanders acclimatize to high altitude more quickly than Caucasians, J Physiol Lond, 2004: 556: 661-671.

19. Wagner PD, A theoretical analysis of factors determining at sea level and altitude, Respir Physiol, 1996: 106: 329-343.

20. Dempsey JA, Hampson PG, Henderson KS, Exercise-induced arterial hypoxaemia in healthy human subjects at sea level, J Physiol Lond, 1984: 355: 161-175.

21. Ferretti G, Moia C, Thomet JM, Kayser B, The decrease of maximal oxygen consumption during hypoxia in man: a mirror image of the oxygen equilibrium curve, J Physiol Lond, 1997: 498: 231-237.

22. West JB, Alexander M, Kellas and the physiological challenge of Mt. Everest, J Appl Physiol, 1987: 63:3-11.

23. Oelz O, Howald H, di Prampero PE, Hoppeler H, Claassen H, Jenni R, Bühlmann A, Ferretti G, Brückner JC, Veicsteinas A, Gussoni M, Cerretelli P, J Appl Physiol, 1986: 60: 1734-1742. 
